\title{
Non Linear Optic in Fiber Bragg Grating
}

\author{
Toto Saktioto ${ }^{1,2}$ and Jalil Ali ${ }^{1}$ \\ ${ }^{1}$ Advanced Photonics and Science Institute, Faculty of Science, \\ Universiti Teknologi Malaysia, Skudai, Johor; \\ ${ }^{2}$ University of Riau, Pekanbaru, Riau; \\ ${ }^{1}$ Malaysia \\ 2Indonesia
}

\section{Introduction}

A Fiber Bragg Grating (FBG) is a periodic variation of the refractive index of the core in the fiber optic along the length of the fiber. The principal property of FBGs is that they reflect light in a narrow bandwidth that is centered about the Bragg wavelength, $\lambda_{B}$ (A. Orthonos and K. Kalli, 1999). FBGs are simple intrinsic devices that are made in the fibre core by imaging an interference pattern through the side of the fibre. They are used as flexible and low cost in-line components to manipulate any part of the optical transmission and reflection spectrum. FBG is formed by the periodic variations of the refractive index in the fiber core. Several techniques have been established to inscribe them with UV-lasers. However, these technologies are limited to photosensitive fiber core material, which are unsuitable for high power applications. Only recently modifications have been demonstrated in a non photosensitive fiber but at the expense of longer exposure times (K. W. Chow et al., 2008). FBGs have all the advantages of an optical fibre, such as electrically passive operation, lightweight, high sensitivity with also unique features for self-referencing and multiplexing capabilities. This gives them a distinct edge over conventional devices (Nahar Singh et. al, 2006, Govind P. Agrawal 2002). Therefore, FBGs in optical fibers have a wide range of applications, such as for sensors, dispersion compensators, optical fibre filters, and all-optical switching and routing (T. Sun et. al,2002). An UV laser source is used to form FBG's in fiber optics either through internal writing or external writing technique (A. Orthonos et al, 1995). The novel idea of using soliton is introduced for FBG.

Solitons are particle-like waves that propagate in dispersive or absorptive media without changing their pulse shapes and can survive after collisions. Various types of optical soliton phenomenon have been studied extensively in the area of nonlinear optical physics. These include the nonlinear Schröedinger solitons in dispersive optical fibers, spatial and vortex solitons in photorefractive material, waveguides and cavity solitons in resonators (Y. S. Kivshar and G. P. Agrawal, 2003).

The principal objective of this topic is to investigate the soliton in FBG showing potential energy. The theory involved in the modelling of soliton is based on the coupled-mode theory including the Kerr nonlinearity, group velocity dispersion (GVD) and self phase modulation (SPM) The motion of a particle moving in FBG represents the pulse propagation in the grating structure of fiber optics exhibiting the existence of optical fiber. In order to describe the photon motion, the function of potential energy is depicted. 


\section{Properties of Fiber Bragg Grating}

A simple form of Fiber Bragg Grating (FBG) in Figure 1 consists of a periodic modulation of the refractive index in the core of a single-mode optical fiber (Phing, H.S.et al, 2008). These types of uniform fiber gratings, where the phase fronts are perpendicular to the fiber longitudinal axis with grating planes have a constant grating period, $\Lambda$.

$$
\lambda_{B}=2 N_{e f f} \Lambda
$$

where $\Lambda$ is the spatial period (or pitch) of the periodic variation and $N_{\text {eff }}$ is the effective index for light propagating in a single mode fiber.

The Bragg condition is a manifestation of both energy and momentum conservation. Energy conservation requires that the frequency of the incident radiation and the reflected radiation is the same, means

$$
\hbar \omega_{f}=\hbar \omega_{i}
$$

Momentum conservation requires that the incident wave vector, $k_{i}$, plus the grating wave vector, $K$, equal the wave vector of the scattered radiation, $k_{f}$. This leads to an equation in which,

$$
k_{i}+K=k_{f}
$$

where the grating wave vector, $K$, has a direction normal to the grating planes with a magnitude $\frac{2 \pi}{\Lambda}$. The diffracted wave vector is equal in magnitude, but opposite in direction to the incident wave vector.

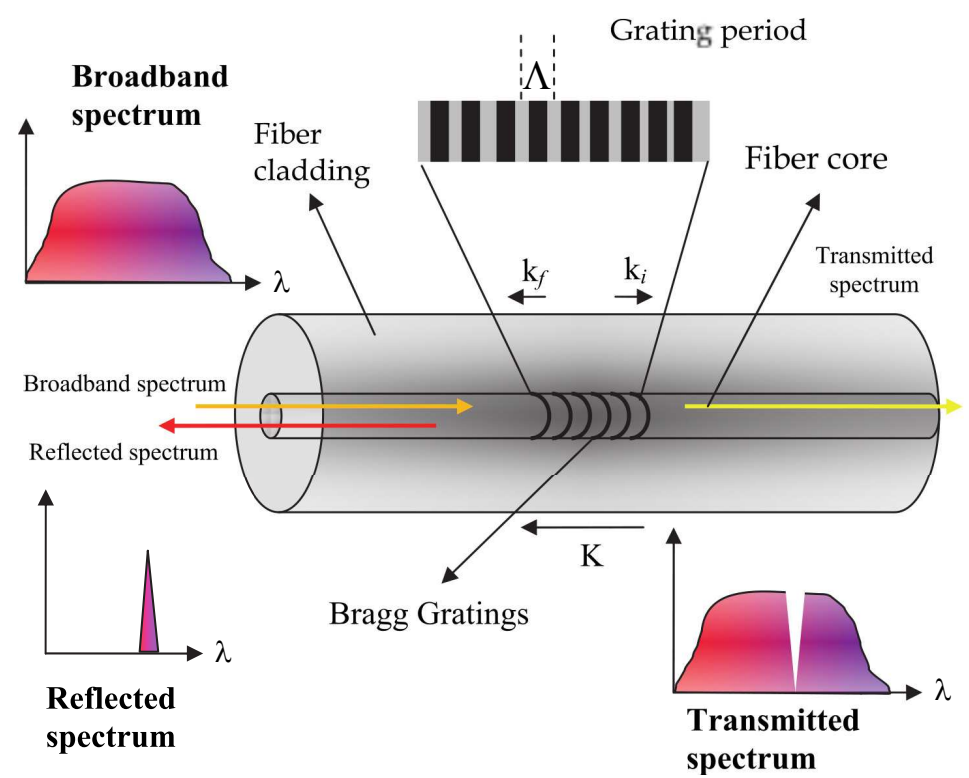

Fig. 1. A basic diagram of Fiber Bragg Grating (A. Orthonos and K. Kalli, 1999). 
Hence, the momentum conservation becomes

$$
2\left(\frac{2 \pi n_{e f f}}{\lambda_{B}}\right)=\frac{2 \pi}{\Lambda}
$$

Equation (4) simplifies to the first-order Bragg condition

$$
\lambda_{B}=2 n_{e f f} \Lambda
$$

$\lambda_{B}$ is the Bragg wavelength. This is the free space center wavelength of the input light that will be back-reflected from the Bragg grating region). $n_{\text {eff }}$ is the effective refractive index of the fiber core at free space center wavelength.

\section{Optical soliton in FBG}

The existence of optical solitons in lossless fiber was theoretically demonstrated first by Hasegawa and Tappert in 1973. Bright and dark solitons appear in anomalous and normal dispersion regime respectively. The existence of an optical soliton in fibers is made by deriving the evolution equation for the complex light wave envelope via the slowly varying Fourier amplitude by retaining the lowest order of the group dispersion. This lower order is taken from the variation of the group velocity as a function of light frequency and the nonlinearity. For a glass fiber it is cubic and originates from the Kerr effect (K. Porsezian, 2007). The one soliton solution of the nonlinear Schrödinger equation is given by a sech $T$ function which is characterized by four parameters, the amplitude, the pulsewidth, the frequency, time position and the phase. In particular, the soliton speed is a parameter independent of the amplitude unlike the case of Kortweg de Vries (KdV) soliton. This is important fact in the use of optical soliton as a digital signal. Originally in 1980, L. F. Mollenauer and his colleagues at Bell Laboratories succeeded in observing optical soliton in fiber. During the 1990's, many other kinds of optical soliton were discovered such as spatiotemporal solitons and quadratic solitons (Y. S. Kivshar and G. P. Agrawal, 2003).

Soliton in fibers is formed after the exact balancing of group velocity dispersion (GVD) arising as a combination of material and waveguide dispersion with that of the self-phase modulation (SPM) due to the Kerr nonlinearity. Due to this, a similar soliton-type pulse formation in Fiber Bragg Grating where the strong grating-induced dispersion is exactly counterbalanced by the Kerr nonlinearity through the SPM and cross-phase modulation (CPM) effects. As a result, there is a formation of slowly travelling localized envelope in FBG structures known as Bragg grating solitons. They are often referred to as gap solitons if their spectra lies well within the frequency of the photonic bandgap if the frequency of incident pulse matches the Bragg frequency. Thus based on the pulse spectrum with respect to the photonic bandgap, solitons in FBG can be classified into two categories as either Bragg grating solitons or gap solitons.

There are basically two conditions that one can determine the formation of solitons in FBG. First is based on high intensity pulse propagation in which the refractive index modulation is weak in FBG where nonlinear coupled-mode (NCM) equations are used to describe a coupling between forward and backward propagating modes. The other conditions deals with the low intensity pulse propagation in FBG where the peak intensity of the pulse is assumed to be small enough so that the nonlinear index change, $n_{2} I$ is much smaller than the 
maximum value of $\delta n$. Under the low intensity limit, the NCM equations can be reduced to the nonlinear Schrödinger equation by using multiple scale analysis.

\section{Coupled-mode theory for FBG}

Several methods have been adopted to study and analyze the reflection and transmission properties of FBG (R. Kasyhap, 2004, M. Liu and P. Shum, 2006). The pulse propagation in FBG and its effect on Bragg grating affect the wave propagation in optical fibers can be examined using the coupled-mode theory (CMT) and Bloch wave technique. However, in this chapter we take CMT only into consideration.

One of the standard methods of analysis of FBG is using the coupled-mode theory (K. Thyagarajan and A. Ghatak and, 2007). According to this theory, the total field at any value of $z$ can be written as a superposition of the two interacting modes and the coupling process results in a $z$-dependent amplitude of the two coupled modes. It is assumed that any point along the grating within the single-mode fiber has a forward propagating mode and a backward propagating mode. Thus the total field within the core of the fiber is given by

$$
\Psi(x, y, z, t)=A(z) \psi(x, y) e^{i(\omega t-\beta z)}+B(z) \psi(x, y) e^{i(\omega t+\beta z)}
$$

where $x, y, z$ refers to space while $t$ refers to variation of time, $A(z)$ and $B(z)$ represents the amplitudes of the forward and backward propagating modes (assumed to be the same order mode), $\psi(x, y)$ represents the transverse modal field distribution, $\omega$ refers to frequency and $\beta$ is the propagation constant of the mode. The total field given by Equation (6) has to satisfy the wave equation given by

$$
\nabla^{2} \Psi+k_{0}^{2} n_{g}^{2}(x, y, z) \Psi=0
$$

where $n_{g}^{2}(x, y, z)$ represents the refractive index variation along the fiber. For an FBG it is given by

$$
n_{g}^{2}(x, y, z)=n^{2}(x, y)+\Delta n^{2}(x, y) \sin (K z)
$$

where $K=2 \pi / \Lambda$ represents the spatial frequency of the grating and $\Delta n^{2}$ represents the index modulation of the grating. For a uniform grating $K$ is independent of $z$; when $K$ depends on $z$, such gratings are referred to as chirped gratings. However, now we further focused on uniform gratings.

Substituting Equation (6) and Equation (7) into Equation (8) and making some simplifying approximations, we can obtain the following coupled-mode equations:

$$
\frac{d A}{d z}=\kappa B e^{i \Gamma z} \text { and } \frac{d B}{d z}=\kappa A e^{-i \Gamma z}
$$

where $\Gamma=2 \beta-K$ and $\kappa$ represents the coupling coefficient given by

$$
\kappa=\frac{\omega \varepsilon_{0}}{8} \iint \psi^{*} \Delta n^{2}(x, y) \psi d x d y
$$




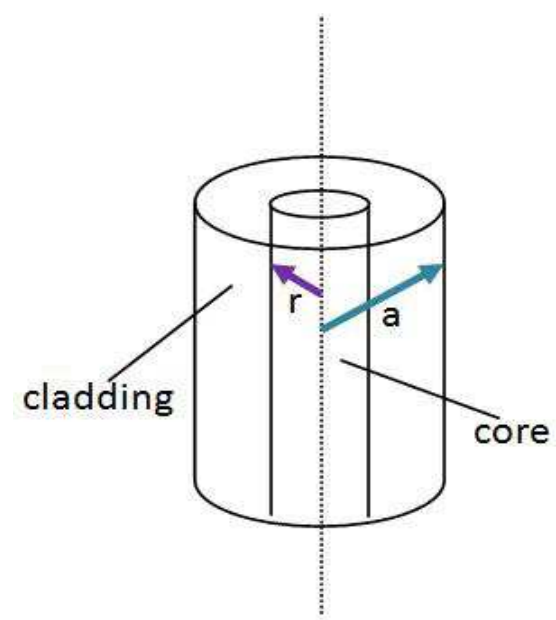

Fig. 2. Cross-section of an optical fiber with the corresponding refractive index profile (R. Kasyhap, 1999).

If the perturbation in the refractive index shown in Figure 2 is constant and finite only within the core of the fiber, then

$$
\begin{aligned}
& \Delta n^{2}(x, y)= \Delta n^{2}, \quad r<a \\
&=0, r>a
\end{aligned}
$$

and we obtain

$$
\kappa \approx \frac{\pi \Delta n}{\lambda_{B}} l
$$

where $\lambda_{B}$ is the Bragg wavelength and

$$
l=\frac{\int_{0}^{a} \psi^{2} r d r}{\int_{0}^{\infty} \psi^{2} r d r}
$$

The coupled-mode Equations (9) can be solved using the boundary conditions of

$$
\mathrm{A}(z=0)=1 \text { and } \mathrm{B}(z=L)=0
$$

where $L$ is the length of the grating. Equation (14) implies that the incident wave has unit amplitude at $\mathrm{z}=0$ and the amplitude of the reflected wave at $z=L$ is zero because there is no reflected wave beyond $z=L$. We defined the reflectivity of the FBG by the ratio of the reflected power at $\mathrm{z}=0$ to the incident power at $z=0$. Solving the coupled-mode equations and using the boundary conditions we obtain the reflectivity of the grating as follows: 


$$
R=\frac{\kappa^{2} \sinh ^{2}(\Omega L)}{\Omega^{2} \cosh ^{2}(\Omega L)+\frac{\Gamma^{2}}{4} \sinh ^{2}(\Omega L)}
$$

where

$$
\Omega^{2}=\kappa^{2}-\frac{\Gamma^{2}}{4}
$$

\section{Pulse propagation in FBG}

Wave propagation in a linear periodic medium has been studied extensively using coupledmode theory. In the case of a dispersive nonlinear medium, the refractive index is given as

$$
\bar{n}(\omega, z, I)=\bar{n}(\omega)+n_{2} I+\delta n_{g}(z)
$$

where $n_{2}$ is the Kerr coefficient and $\delta n_{g}(\mathrm{z})$ accounts for periodic index variation inside the grating. The coupled-mode theory can be generalized to include the nonlinear effects if the nonlinear index change, $n_{2} I$ in Equation (2.11) is so small that it can be treated as a perturbation.

The starting point consists of solving Maxwell's equations with the refractive index given in Equation (16). When the nonlinear effects are relatively weak, we can work in the frequency domain and solve the Helmholtz equation,

$$
\nabla^{2} \tilde{E}+\tilde{n}^{2}(\omega, z) \omega^{2} / c^{2} \tilde{E}=0
$$

The forward and backward propagating modes in FBG due to Bragg reflection can be described using CMT as been explained by Yariv in the distributed feedback structure (K. Senthilnathan, 2003). As usual, the governing equations for the pulse propagation in FBG are derived using Maxwell's equation. In this study the focus is on the frequency domain as the nonlinear effects are assumed to be relatively weak. It can easily be shown that Maxwell's equation are reduced to the following wave equation in the form

$$
\frac{\partial^{2} \vec{E}}{d z^{2}}-\frac{\varepsilon(z)}{c^{2}} \frac{\partial^{2} E}{\partial t^{2}}=0
$$

where perturbed permittivity, $\varepsilon(z)=\bar{n}^{2}+\tilde{\varepsilon}(z), \bar{n}^{2}$ is the spatial average of $\tilde{\varepsilon}(z)$, and $\bar{n}$ is the average refractive index of the medium. We consider the term $\tilde{\varepsilon}(z)$ with a period $\Lambda$ and define $k_{0}=\pi / \Lambda$. Using the Fourier series, $\tilde{\varepsilon}(z)$ can be written as

$$
\tilde{\varepsilon}(z)=2 \tilde{\varepsilon} \cos \left(2 k_{0} z\right)
$$

This electric field inside the grating can be written as

$$
\vec{E}(z, t)=\vec{E}_{f}(z, t) e^{+i\left(k_{b} z-\omega_{d} t\right)}+\vec{E}_{b}(z, t) e^{-i\left(k_{a} z-\omega_{a} t\right)}+\ldots
$$

where $E_{f, b}(z, t)$ represents the forward and backward propagating waves, respectively, inside the FBG structure. Now, inserting Equation (19) and (20) into Equation (18) and 
considering that the fields $E_{f, b}(z, t)$ are varying slowly with respect to $\omega_{0}^{-1}$ in time and $k_{0}^{-1}$ in space, the resulting frequency domain coupled mode equations can be written as

$$
\begin{aligned}
& i \frac{\partial \vec{E}_{f}}{\partial z}+i \frac{\bar{n}}{c} \frac{\partial \vec{E}_{f}}{\partial t}+\kappa \vec{E}_{b}=0 \\
& -i \frac{\partial \vec{E}_{b}}{\partial z}+i \frac{n}{c} \frac{\partial \vec{E}_{b}}{\partial t}+\kappa \vec{E}_{f}=0
\end{aligned}
$$

The value of $\kappa$ represents the coupling between the forward and backward propagating waves in the FBG. The set of Equations (21) are called linear coupled-mode (LCM) equations in which the non-phase-matched terms have been neglected. The LCM equations assume slowly varying amplitudes rather than the electric field itself. Note that CMT is an approximate description that is valid for shallow gratings and for wavelength close to the Bragg resonance.

\section{Potential energy distribution in FBG}

In the presence of Kerr nonlinearity, using CMT, the NLCM equations can be written as

$$
\begin{aligned}
& i \frac{\partial \vec{E}_{f}}{\partial z}+i \frac{\bar{n}}{c} \frac{\partial \vec{E}_{f}}{\partial t}+\kappa \vec{E}_{b}+\left(\Gamma_{s}\left|\vec{E}_{f}^{2}\right|+2 \Gamma_{x}\left|\vec{E}_{b}^{2}\right|\right) \vec{E}_{f}=0 \\
& -i \frac{\partial \vec{E}_{b}}{\partial z}+i \frac{\bar{n}}{c} \frac{\partial \vec{E}_{b}}{\partial t}+\kappa \vec{E}_{f}+\left(\Gamma_{s}\left|\vec{E}_{b}^{2}\right|+2 \Gamma_{x}\left|\vec{E}_{f}^{2}\right|\right) \vec{E}_{b}=0
\end{aligned}
$$

where $E_{f}$ and $E_{b}$ are the slowly varying amplitudes of forward and backward propagating waves, $\bar{n}$ is the average refractive index, and $\Gamma_{\mathrm{s}}$ and $\Gamma_{\mathrm{x}}$ are SPM and Cross-Phase modulation terms. In Equation (22) the material and waveguide dispersive effects are not included due to the dispersion arising from the periodic structures dominates the rest near Bragg resonance condition. Noted that the above NLCM equations are valid only for wavelengths close to the Bragg wavelength.

Now, by substituting the stationary solution to the above coupled-mode equations is by assuming

$$
E_{(f, b)}(z, t)=e_{(f, b)}(z) e^{-i \hat{\delta} c t / \bar{n}}
$$

where $\hat{\delta}$ is the detuning parameter. Using the stationary solution in Equation (22), we obtain

$$
\begin{aligned}
& i \frac{d e_{f}}{d z}+\hat{\delta} e_{f}+\kappa e_{b}+\left(\Gamma_{s}\left|e_{f}\right|^{2}+2 \Gamma_{x}\left|e_{b}\right|^{2}\right) e_{f}=0 \\
& -i \frac{d e_{b}}{d z}+\hat{\delta} e_{b}+\kappa e_{f}+\left(\Gamma_{s}\left|e_{b}\right|^{2}+2 \Gamma_{x}\left|e_{f}\right|^{2}\right) e_{b}=0
\end{aligned}
$$


Equation (24) represents the time-independent light transmission through the grating structure, where $e_{f}$ and $e_{b}$ are the forward and backward propagating modes $\kappa$ represents $n_{0 k}, \quad\left(n_{0 k}=\frac{n_{01}-n_{02}}{\pi}\right)$ where $n_{01}$ is the core refractive index and $n_{02}$ is the cladding refractive respectively, $\Gamma_{s}$ represents Self Phase Modulation and $\Gamma_{x}$ represents Cross-phase modulation effects. This has been extensively investigated by many researchers. The NLCM equations are non-integrable in general. But in a few cases, NLCM equations have exact analytical solutions representing the solitary wave solutions. However, Christoudolides and Joseph have obtained the soliton solution to the NLCM equation, known as slow Bragg soliton, under the integrable massive Thirring model where the SPM and detuning parameter is set to zero. After using suitable transformation, it is used in nonlinear optics as a simple model to explain the self-induced transparency effect. Using the Stokes parameters they derived the relation of energy density for the stationary solution for the NLCM equation in terms of the Jacobi elliptic function.

There are some possible interesting soliton-like solutions apart from these stationary solutions. In the fiber Bragg grating, these soliton-like solution for the NLCM equations carry a lot of practical importance.

\section{Solution of optical soliton using NLCM}

Wave propagation in optical fibers is analyzed by solving Maxwell's Equation with appropriate boundary conditions. In the presence of Kerr nonlinearity, using the coupledmode theory, the nonlinear coupled mode equation is defined under the absence of material and waveguide dispersive effects. The dispersion arising from the periodic structure dominates near Bragg resonance conditions and it is valid only for wavelengths close to the Bragg wavelength.

In order to explain the formation of Bragg soliton, consider the Stokes parameter since it will provide useful information about the total energy and energy difference between the forward and backward propagating modes. In this study, the following Stokes parameter are considered where

$$
\begin{aligned}
& A_{0}=\left|e_{f}\right|^{2}+\left|e_{b}\right|^{2}, \\
& A_{1}=e_{f} e_{b}^{*}+e_{f}^{*} e_{b}, \\
& A_{2}=i\left(e_{f} e_{b}^{*}-e_{f}^{*} e_{b}\right) \\
& A_{3}=\left|e_{f}\right|^{2}-\left|e_{b}\right|^{2}
\end{aligned}
$$

with the constraint $A_{0}^{2}$ equals to the sum of $A_{1}^{2}+A_{2}^{2}+A_{3}^{2}$. In the FBG theory, the nonlinear coupled-mode (NLCM) equation requires that the total power $P_{0}=A_{3}=\left|e_{f}\right|^{2}-\left|e_{b}\right|^{2}$ inside the grating is constant along the grating structures. Rewriting the NLCM equations in terms of Stokes parameter gives

$$
\frac{d A_{0}}{d z}=-2 \kappa A_{2}, \quad \frac{d A_{1}}{d z}=2 \hat{\delta} A_{2}+3 \Gamma A_{0} A_{2}^{\prime}
$$




$$
\frac{d A_{2}}{d z}=-2 \hat{\delta} A_{1}-2 \kappa A_{0}-3 \Gamma A_{0} A_{1}, \frac{d A_{3}}{d z}=0
$$

In Equation (26), we drop the distinction between the SPM and cross modulation effects. Hence Equation (26) becomes $3 \Gamma=2 \Gamma_{x}+\Gamma_{s}$. It can be clearly shown that the total power, $P_{0}$ $\left(=A_{3}\right)$ inside the grating and is found to be constant meaning it is conserved along the grating structure. In the derivation of the anharmonic oscillator type equation, it is necessary to use the conserved quantity. This is obtained in the form $\hat{\delta} A_{0}+\frac{3}{4} \Gamma A_{0}^{2}+\kappa A_{1}=C$, where $C$ is the constant of integration and $\hat{\delta}$ is the detuning parameter. Equation (27) can further be simplified to (Yupapin, P.P. et al, 2010),

$$
\frac{d^{2} A_{0}}{d z^{2}}-\alpha A_{0}+\beta A_{0}^{2}+\gamma A_{0}^{3}=4 \hat{\delta} C
$$

where $\alpha=2\left[2 \hat{\delta}^{2}-2 \kappa^{2}-3 \Gamma C\right], \beta=9 \Gamma \hat{\delta}$ and $\gamma=\frac{9}{4} \Gamma^{2}$. Equation (27) contains all the physical parameter of the NLCM equation. Physically, a represents the function of detuning parameters, and phase modulation factors (SPM and CPM). $\beta$ represents the function of phase modulation factors (SPM and CPM) and the detuning parameters. Lastly, $\gamma$ represents the phase modulation factor (SPM and CPM). In general, $a, \beta$ and $\gamma$ are the oscillation factors.

\section{Potential energy distribution in FBG structures}

In order to describe the motion of a particle moving within a classical anharmonic potential, we have the solution of Equation 28 in the form of

$$
V\left(A_{0}\right)=-\alpha \frac{A_{0}^{2}}{2}+\beta \frac{A_{0}^{3}}{3}+\gamma \frac{A_{0}^{4}}{4}
$$

It represents the potential energy distribution in a FBG structures while the light is propagating through the grating structures.

In Equation (28), $\beta$ is not considered due to power conservation along the propagating of this FBG structure. The qualitative aspects of the potential well will change if the nonlinearity parameter of the wave equation is varied.

Figure 3 depicts the double-well potential under Bragg resonance condition where $\beta=0, \gamma=$ 0.23 and $\alpha$ is varied from 0.1 to 1.0. Photon with power of less than the total power, $P_{0}$ will only travel inside the well unless their energy exceeds the energy level. This would allow the photon to move outside the well.

Figure 4 explains the optimized point for various values of $\alpha$. The graph clearly shows that the optimized points decreased exponentially when values of $\alpha$ are increased. However, when $\alpha>>1$, the trend of the curve is no longer valid since it turns into an almost linear relationship.

Figure 5 shows the motion of photon in double well potential under different values of gamma for the Bragg resonance condition of $\gamma$ from 0.13 to 0.53 . Note that the increment of gamma which is between $0.53<\gamma<1$ will reduce the double well potential to a single well potential. 


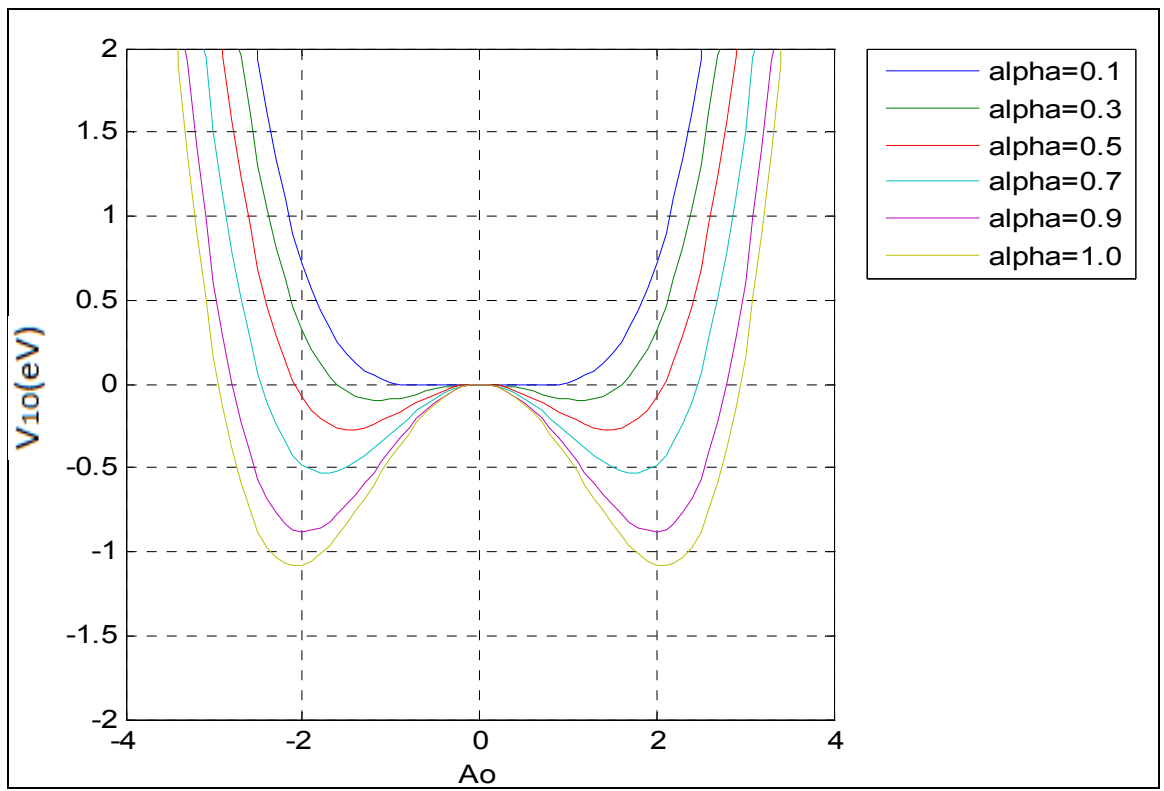

Fig. 3. The motion of photon in double well for different values of $\alpha$.

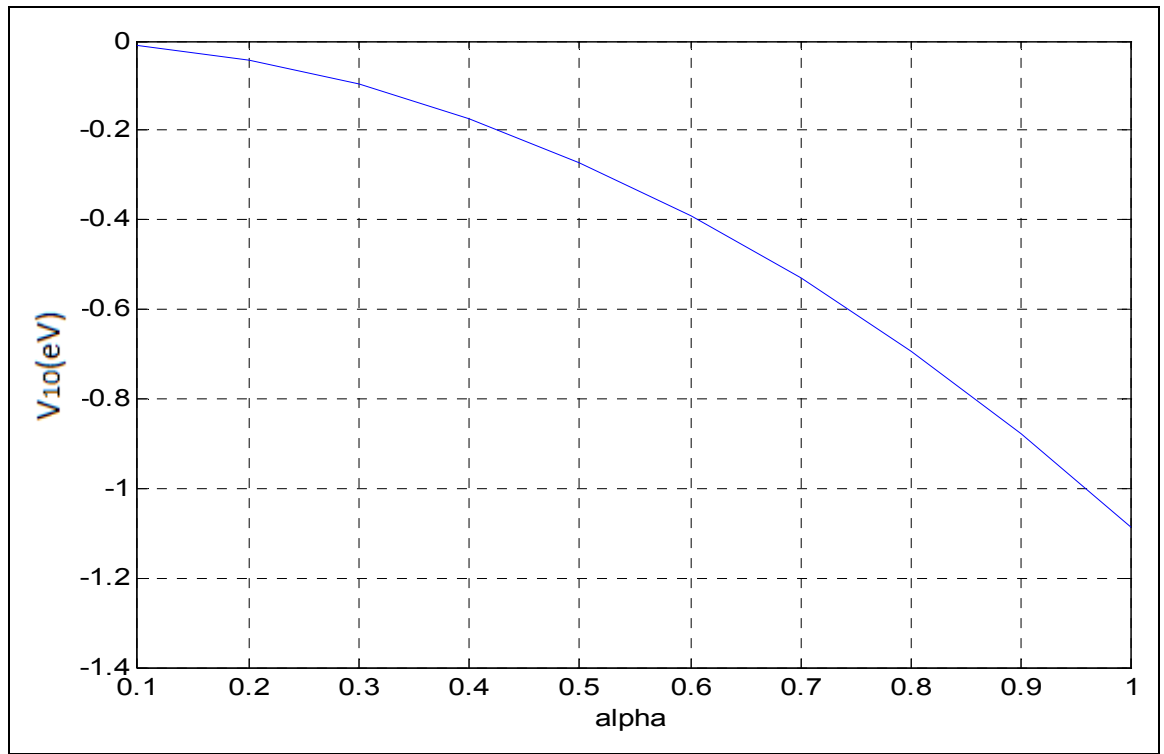

Fig. 4. The optimized point of the double well potential for different values of $\alpha$. 


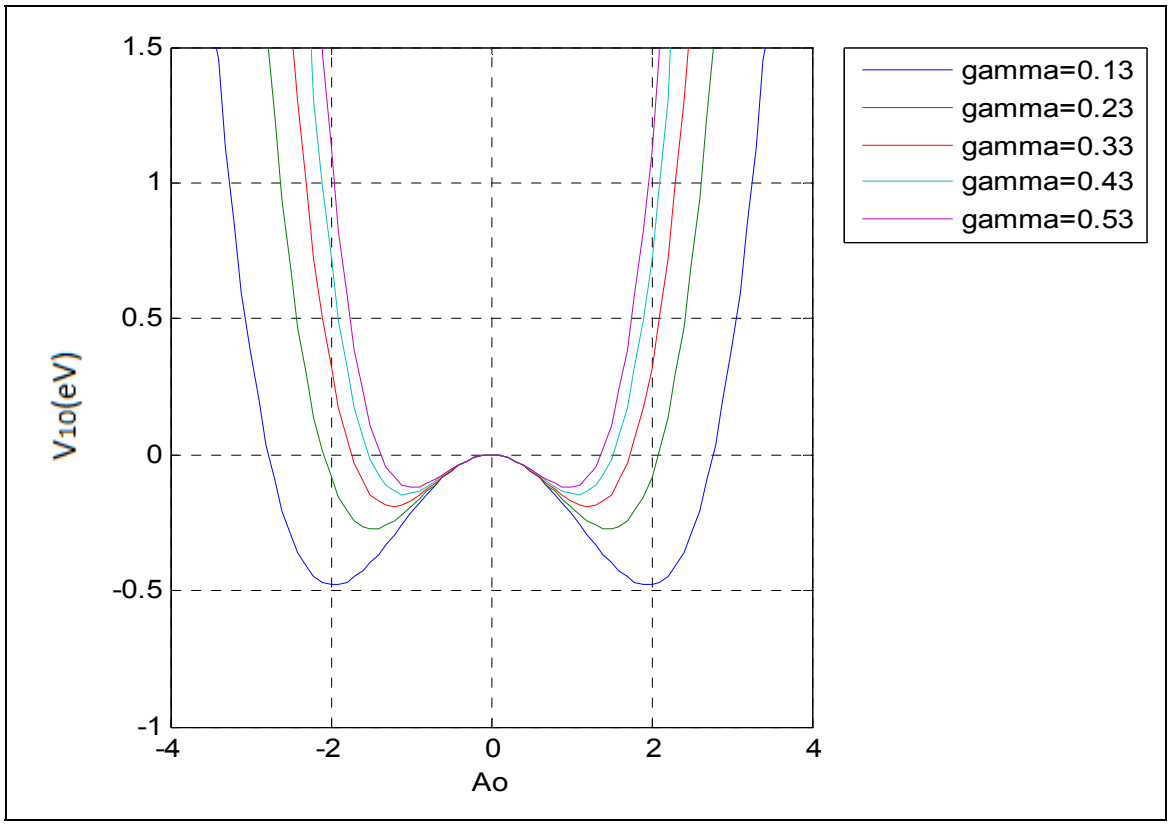

Fig. 5. Under Bragg resonance condition the system possesses double well potential for $\gamma$ 0.13 to 0.53 .

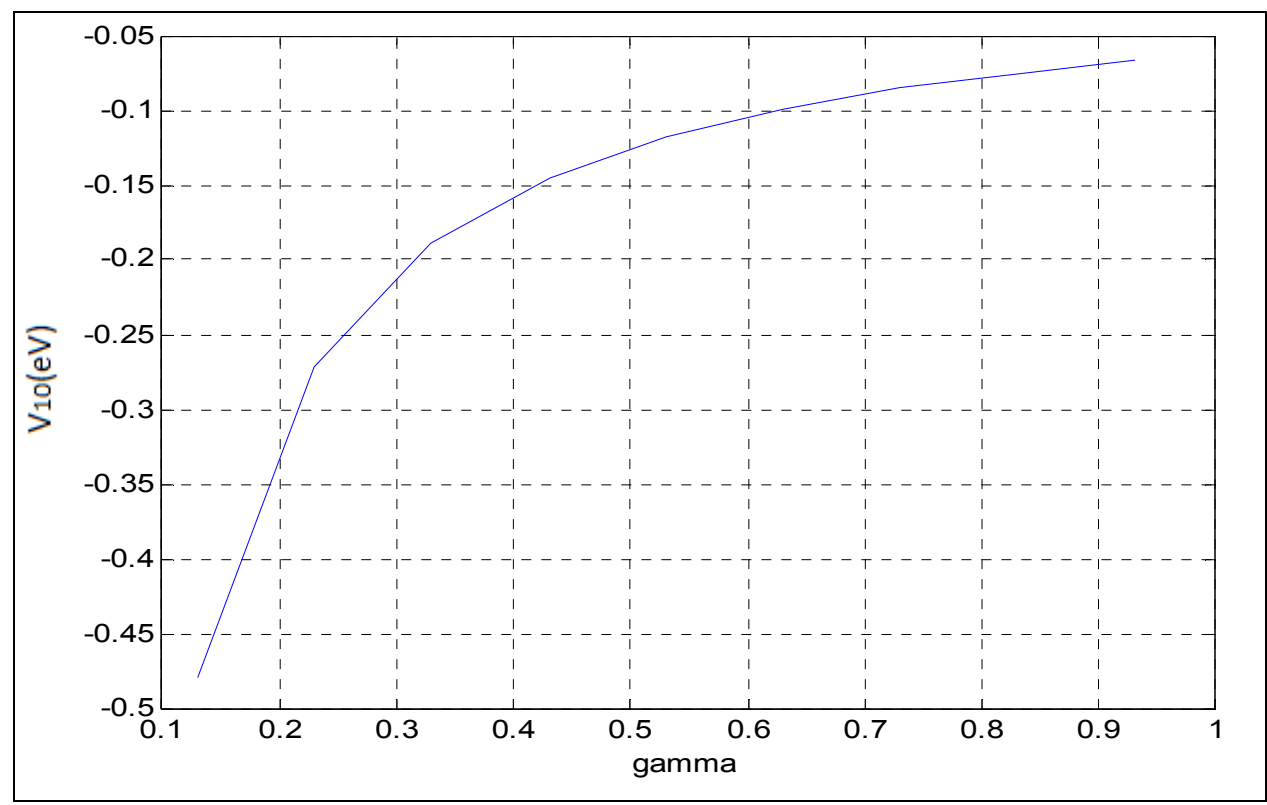

Fig. 6. The optimized point of the double well potential when $\gamma=0.1$ to 1.0. 
Figure 6 describes the optimized point for varies of gamma, $\gamma$. Parametric variation of gamma produces a potential energy function which increases exponentially. However, when $\gamma>>1$, a plateau is observed. This shows that it is not valid if $\gamma \rightarrow \infty$.

\section{External disturbance of potential energy photon in FBG}

By using Equation (28) where another term is considered, then we can have

$$
\frac{d^{2} A_{0}}{d z^{2}}-\alpha A_{0}+\beta A_{0}^{2}+\gamma A_{0}^{3}+\theta A_{0}^{4}=4 \hat{\delta} C
$$

where $\alpha=2\left[2 \hat{\delta}^{2}-2 \kappa^{2}-3 \Gamma C\right], \beta=9 \Gamma \hat{\delta}, \gamma=\frac{9}{4} \Gamma^{2}$ and $\theta=f(\theta)$. To simplify Equation

(29), it is assumed the parameters of $\alpha, \beta$ and $\gamma$ is independent with respect to parameter $\theta$. Equation (29) contains all the physical parameter of the NLCM equation.

In order to describe the motion of a particle moving with the classic anharmonic potential, where the external disturbance is involved then we have the solution as follows,

$$
V\left(A_{0}\right)=-\alpha \frac{A_{0}^{2}}{2}+\beta \frac{A_{0}^{3}}{3}+\gamma \frac{A_{0}^{4}}{4}+\theta \frac{A_{0}^{5}}{5}
$$

It represents the potential energy distribution in the Fiber Bragg Grating structures.

Figure 7 depicts the motion of photon in a potential well which changes when few nonlinear parameters are taken into account as shown is Equation (30). Photon is trapped by the $\alpha$ parameter which is depicted by legend $\mathrm{V}$. When $\alpha$ is too large, the potential well produces $A_{0}$ increases and have a wider of double well. The $\gamma$ parameter is shown by $\mathrm{X}$ legend. When $\gamma$ is large, the potential well produces $A_{0}$ increases. Suppose that the source is imposed to FBG than initial power is used to generate the particles. It shows that double well potential well is not symmetrical and the potential energy will decrease at certain region and is shown in Figure 7 in legend $Y$. The other effect is the disturbance at potential energy by legend $Z$ where photon cannot be trapped symmetrically. It will tend to equilibrium but it is not stable where the photon leaves the potential curve as a losses.

In terms of parametric function, we can describe it as follows. The change in $\alpha$ will affect the dip of the potential well. If $\alpha$ is approximately too small, the shape of the potential well develop into a single potential well. The occurrence of $\beta$ effect in the motion of photon gives an effect to the negative region which means $A_{0}<0$. The effect of $\gamma$ also shows that the width of potential well will decrease if we increase the value of $\gamma$. Therefore if we increase the value of gamma, we can assume that the photon will be localized and can be trapped. In addition, another nonlinear factor $\theta$, it will change the shape of potential well rapidly. We could say that if we include the existence of $\theta$, the shape of potential well becomes chaotic. The photon does not only move within a certain region that is known as the potential well and moving freely.

Figure 8 shows the effect of external disturbance, $\theta$. It shows that by increasing the value of $\theta$, it will also affect the change in $\gamma$. In other words, the negative part of Ao will be influenced it potential energy. The different values of $\gamma$ will produce different profiles. By simulating, we assumed that the increased of $\gamma$ value from 0.3 to 0.9 , the curve will be positioned within the region $C$. The peak of $V$ for each $\gamma$ from 0.3 to 0.9 describes $\theta$ increases linearly and large gradient compare to the initial $\mathrm{V}$. This represents that potential energy cannot maintain photon to be trapped and equilibrium state if $\gamma$ is relatively small. 


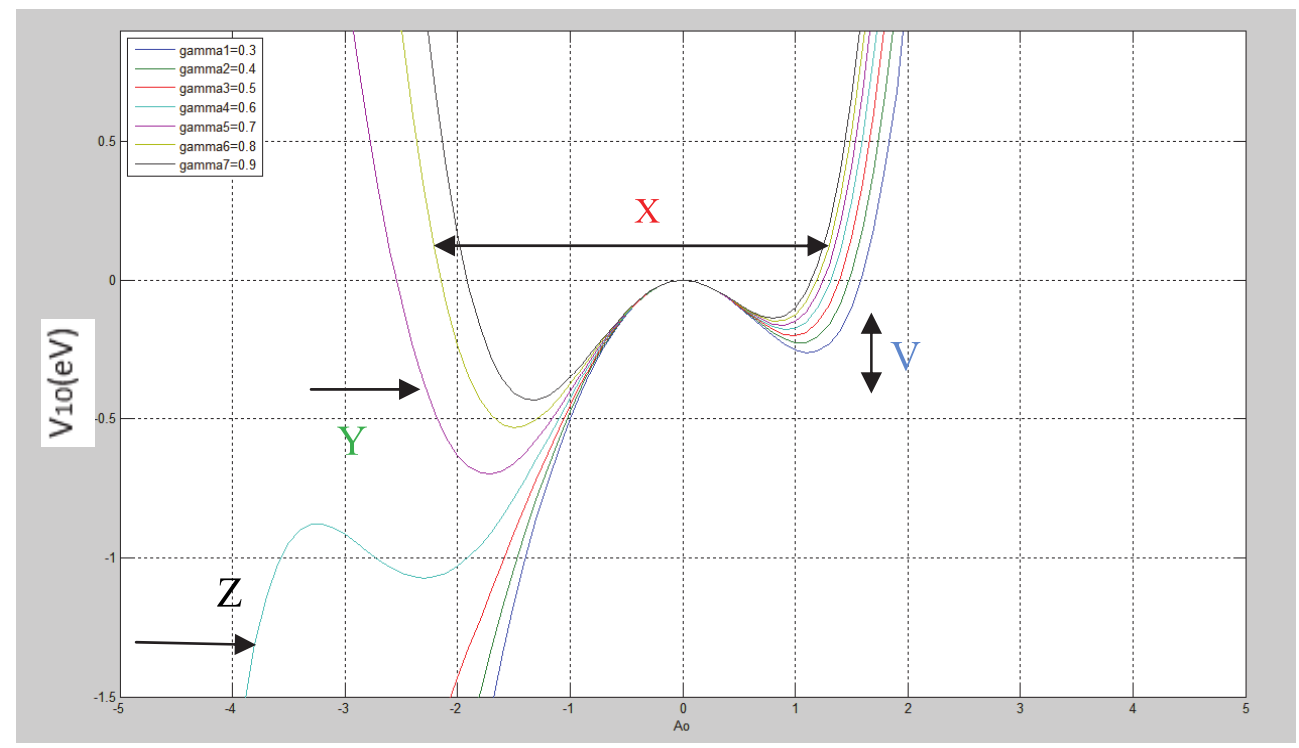

Fig. 7. The motion of photon in potential well for $a=0.9, \beta=0.3, \theta=0.09$ and $\gamma$ is varies from 0.3 to 0.9 .

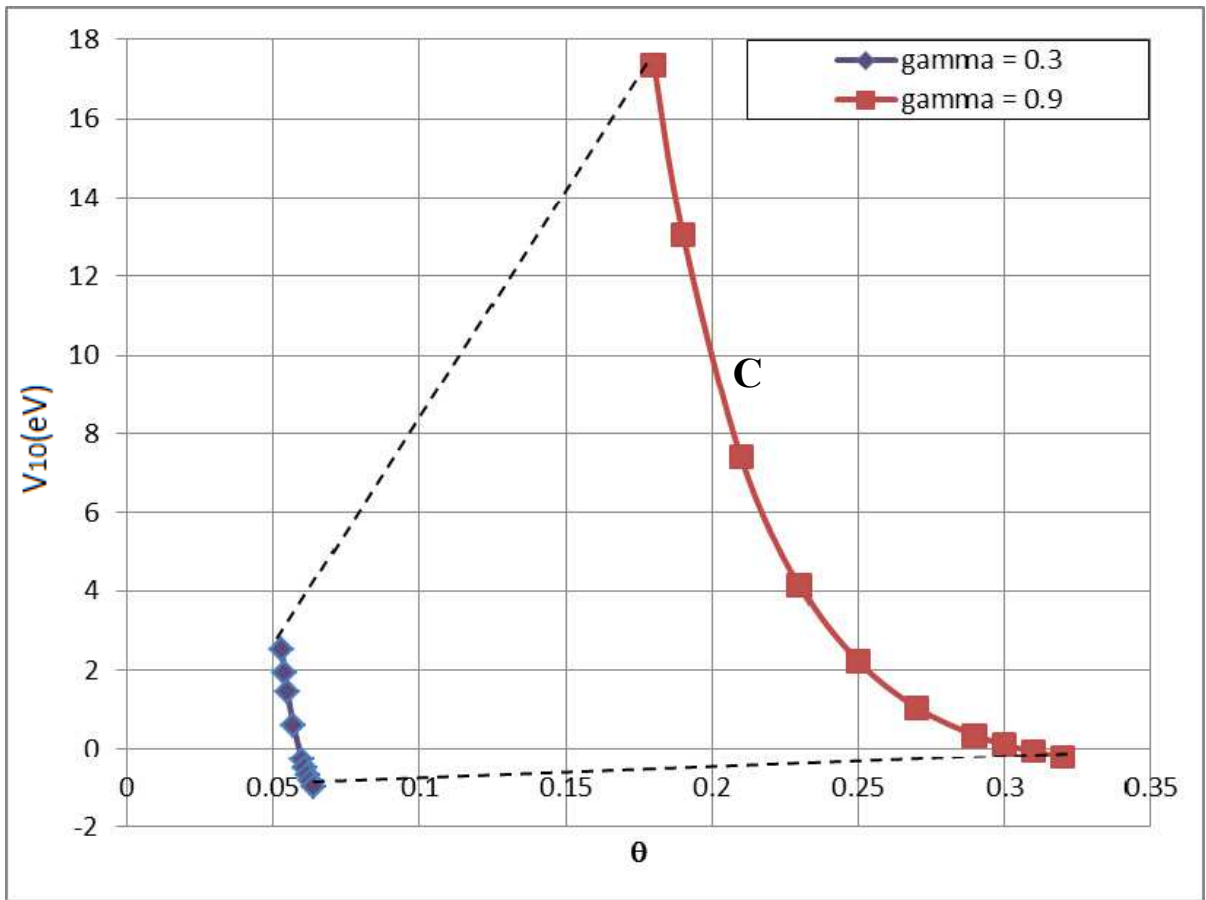

Fig. 8. The effect of theta, $\theta$ to $\gamma$ and shape of the potential well of the photon. 
In Figure 9 it can be shown that by increasing the value of $\beta$ the potential energy of the potential well will be reduced. The highest potential drop occurs within the range of $\beta, 0.2$ to 0.3. If the disturbance is large, it requires a high potential energy to maintain the photon especially for $\gamma=0.7$. In other words, increasing the $\gamma$ value will affect the shape of the potential well in terms of the potential energy. It will affect the equilibrium of the potential well and therefore the trapped photons are no longer being trapped or localized.

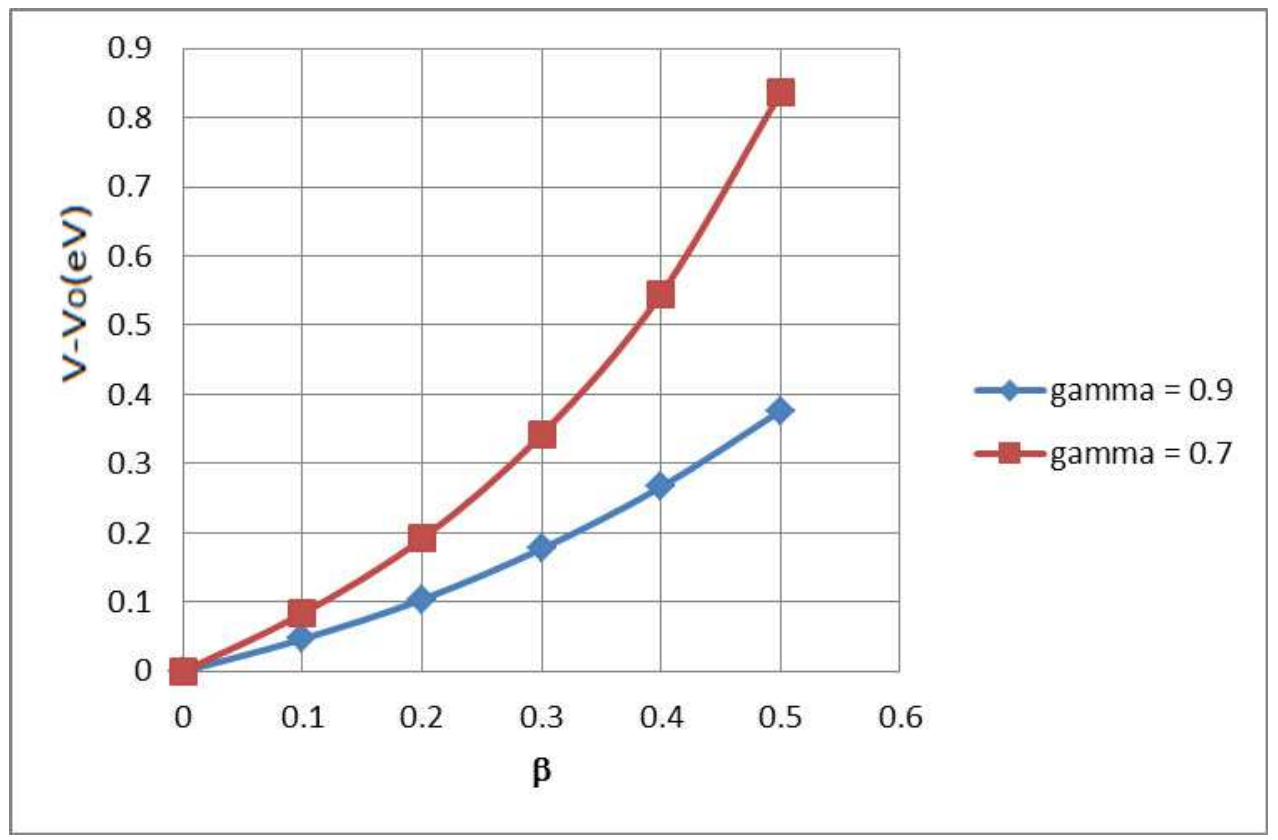

Fig. 9. The disturbance to the potential energy by $\beta$ factor.

\section{Photon due to external energy perturbation in potential well}

Figure 10 depicts the motion of photon in potential well which changes when few nonlinear parameters is take into account as described by Equation (30). There are theoretically some comments in this figure. Photon is trapped by $\alpha$ parameter which is depicted by legend V. When $\alpha$ is too large, the potential well produces $A_{0}$ increases and have a wider double well. The value of $\gamma$ parameter is shown by $\mathrm{X}$ legend. When $\gamma$ is large, the potential well produces 
$A_{0}$ increases. Suppose that the source is imposed to FBG than initial power is used to generate the particles. It shows that double well potential well is not symmetric and potential energy will decrease at the certain region in legend $Y$. The other effect is the perturbation of potential energy by legend $\mathrm{Z}$ where photon cannot be trapped symmetrically. It will tend to equilibrium but it is not stable where it can go for losses.

The change in the parametric function can be easily described in terms of $a, \beta$ and $\gamma$. The dip in the potential well will transform with a single potential well when $a$ is extremely small. $\beta$ affects the photon motion which in turn will effect to the negative region of the potential well when $A_{0}<0$.The effect of $\gamma$ shows that the width of potential well will decrease if we increased the value of $\gamma$. The photon will be trapped when $\gamma$ is increased. The shape of the potential well can be controlled by a nonlinear factor $\theta$. The changes in the value of $\theta$ lead to a chaotic behaviour of the potential well. Under these conditions the photon can either move within certain specific regions or act as a free particle. Thus, Figure 10 illustrates the single perturbation as described by the nonlinear parameter, $\theta$.

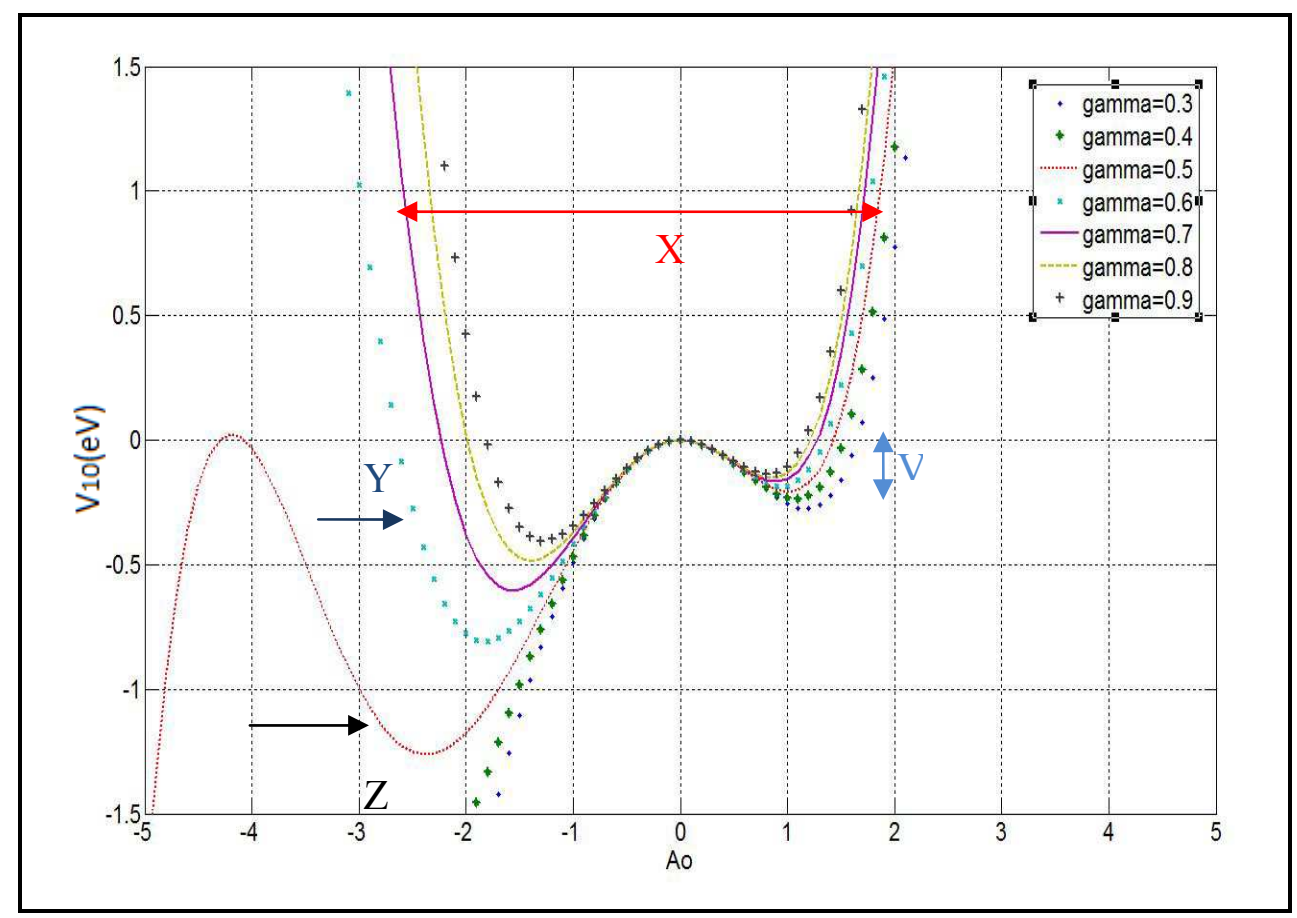

Fig. 10. The motion of photon in potential well for $a=0.9, \beta=0.3, \theta=0.09$ and $\gamma$ is varies from 0.3 to 0.9 . 
Now consider the case in Equation (27) with a set of constraints which is governed by $\phi_{(e)}=\sum_{n=0}^{\infty} A_{0}{ }^{n}$. The perturbation factor then is

$$
\frac{d^{2} A_{0}}{d z^{2}}=\left.\phi_{e}^{\prime \prime}\right|_{n=0}
$$

If Equation (30) is accumulated using the external perturbation then

$$
\left.\phi^{\prime \prime}\right|_{n=0}+\sum_{\substack{n=0 \\ m=1}}^{\infty} C_{m} A_{0}{ }^{n}=\psi
$$

where $\psi$ is a function of $f\left(\delta, C, C_{m}\right)$ and $C_{m}=[\alpha, \beta, \gamma, \ldots]$

The value of $m=2 n$ for $n=1,2,3, \ldots, m=2 n+1$ for $n=0,1,2, \ldots$

$C$ is constant and $C=\left(C_{1}, C_{2}, C_{3}, \ldots, C_{m}\right)$. The value of $C$ is linear to $A_{0}$ but not to $V$. Equation (31) can then be modified by

$$
V\left(A_{0}\right)=\sum_{\substack{m=1 \\ n=0}}^{\infty} C_{m} A_{0}^{n}
$$

Equation (32) represents the complete potential energy distribution in the Fiber Bragg Grating structure. We believe at this juncture, the potential function is modified from Conti and Mills (C. Conti and S. Trillo, 2001). Using well-known Duffing oscillator type equation, analogically it is written as

$$
\phi_{e}{ }^{\prime \prime}+\sum_{\substack{m=1 \\ n=0}}^{\infty} C_{m} A_{0}{ }^{n}=0
$$

For multi perturbation of nonlinear parameters, two major shapes will be simplified in the series term. The coupled mode equations are solved under different conditions when soliton is used for FBG writing. The cases examined are (i) when there is no energy disturbance (ii) the effect of potential energy disturbance factor (iii) potential energy with the highest disturbance factor.

When multi perturbations are considered then the photon will be trapped and untrapped for various conditions. As depicted in Figure 11, it explains the extrapolation of the graph if more factors of perturbation added into Equation (32). The addition of parametric factors by the higher odd number, Figure 11 (a) will allow the photon to move in a well, and Figure 11 (b) will lead the photon to be untrapped and higher even number. It is clearly shown in the graphs that as $n>>\infty$, the value of $\left|A_{0}\right|$ will remain constant in the range of $-2<A_{0}<2$. However, when the value of $V_{(0)}$ is equal to zero, there are many possibilities of $A_{0}$, meaning the exact value of intentsity, $A_{0}$ to trap the photon is difficult to determine in this condition. If the parametric factor considered is too large then we may conclude that the photon is in indifferent state part of the equlibrium. 


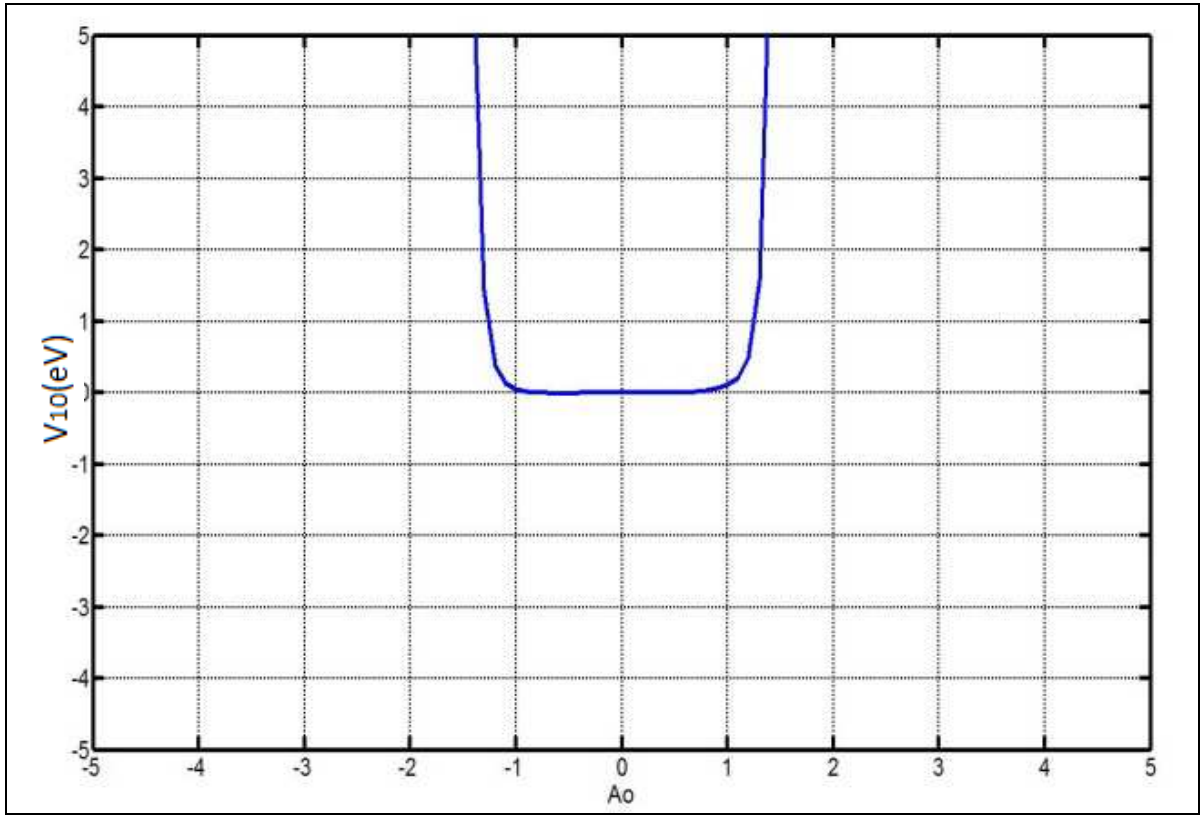

(a)

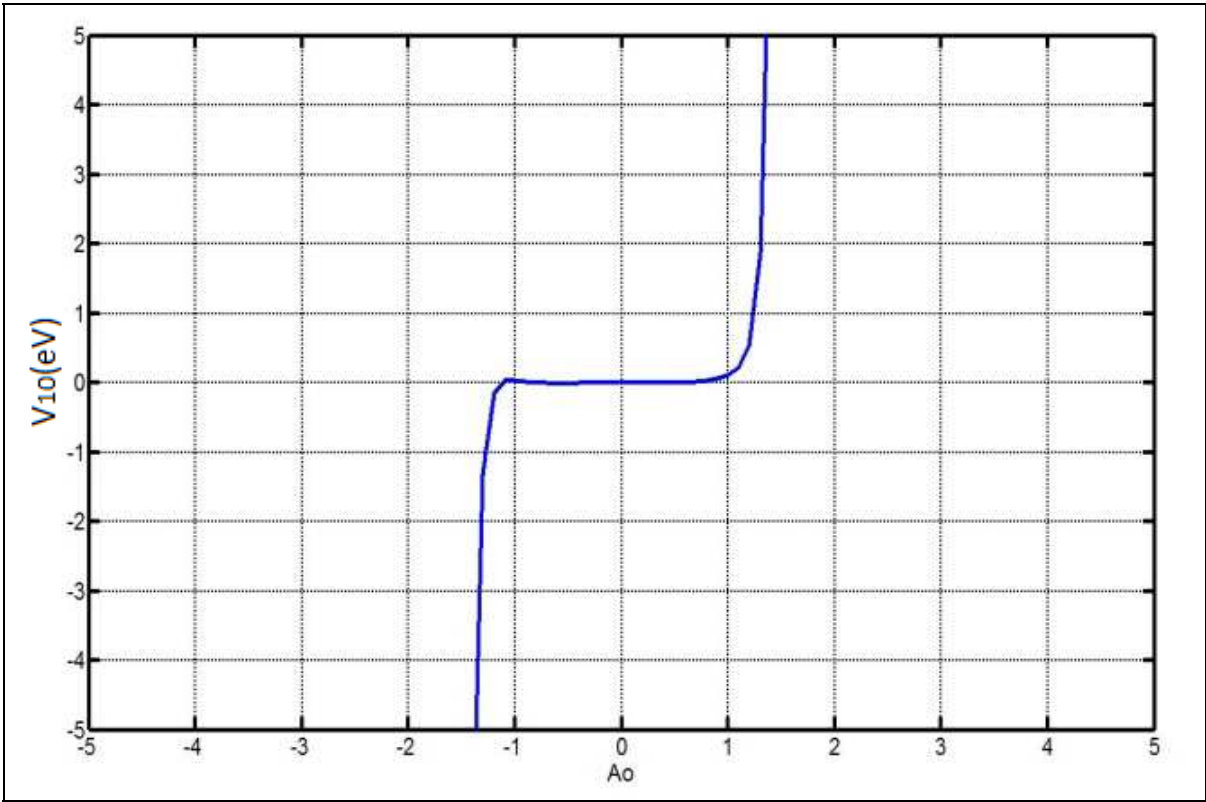

(b)

Fig. 11. The disturbance factor that affect the shape of the potential well of the motion of photon. 
The stationary solutions of Equation (32) are applied neither for bright nor dark soliton solution since the dominant parametric factor in contributing $A_{0}$ is unknown. However, from Equation (32) we have

$$
A_{0}=f\left(C_{m}, z\right)
$$

Under these conditions, the frequencies with photonic band gap keep forming an envelope after the exact balancing at grating-induced dispersion with nonlinearity. Either decay or increase, the forward and backward waves are transferred by Bragg reflection process. The total energy of the system, potential energy function is equal to zero having multi perturbation which is $-1<A_{0}<1$ and if $V \rightarrow \infty, A_{0}=2$.

\section{Conclusion}

The novel idea of using soliton in Fiber Bragg Gratings (FBG) shows that the motion of a particle moving in FBG represents the pulse propagation in the grating structure of FBG. This indicates the existence of optical soliton. It is described in terms of the photon motion and as a function of potential energy. Results obtained show that photon can be trapped by nonlinear parameters of potential energy which are identified as $\alpha, \beta, \gamma$ and $\theta$.

In the first simulation results of nonlinear parametric studies of photon in a FBG, we have successfully shown that the changes of nonlinearity parameter will affect the motion in the potential well. This will influence the existence of Bragg soliton in a fiber Bragg grating. In the second simulation results, we have added new nonlinear parameter which is known as $\theta$. We have preset the value of $\theta, \alpha$ and $\beta$ and vary the value of $\gamma$ over certain range. From the results, it is depicted that the factor $\theta$ will affect the shapes of potential well. If the existence of $\theta$ is taken into account, the potential well profile becomes chaotic.

The simulation data are then expanded on the multi perturbation of potential energy photon in FBG. It shows that the change of $a$ affect the dip of the potential well. The occurrence of $\beta$ effect in the motion will affect the soliton propagation in the region for $A_{0}<0$. The effect of $\gamma$ shows that the width of potential well will decreased if the value of $\gamma$ is increased. However, another nonlinear factor, $\theta$ will turns the shape of potential well rapidly which necessities the multi perturbation studies. When multi perturbations are considered, the photon will be trapped and entrapped under various conditions. From this, we may conclude the addition of nonlinear parametric factors by the higher odd number will allow the photon to move in a well instead to be entrapped with the higher even number. It is found from this study that the potential well under Bragg resonance condition is not symmetrical and conserved. The higher perturbation series representing the potential well is much indifferent of the equilibrium in both odd and even nonlinear parametric factor of $n$.

As a conclusion, these studies have successfully shown that it is plausible to use soliton for FBG writing and the soliton can be controlled by manipulating the parametric effects which are $\alpha, \beta, \gamma$ and $\theta$. The model developed in this topic can be further extended by optimizing the nonlinear parameters in terms of the potential energy, soliton trapping and 
its applications as optical tweezers. The model can be tested by developing compact miniature FBG inscribing system using laser diode.

\section{Acknowledgment}

We would like to thank the Institute of Advanced Photonic Science, Faculty of Science, Universiti Teknologi Malaysia (UTM) and Physics Department, Faculty of Math and Natural Sciences, University of Riau, Pekanbaru, Indoneseia for generous support in this research.

\section{References}

Andreas Orthonos and Kyriacos Kalli. (1999).“Fiber Bragg Gratings - Telecommunications and Sensing". Artech House Boston, London.

Andreas Orthonos and Xavier Lee. "Novel and Improved Methods of Writing Bragg Gratings with Phase Masks". IEEE Photonics Technology Letters, Vol. 7, No. 10, October 1995.

C. Conti and S. Trillo, "Bifurcation of gap solitons through catastrophe theory". Phys. Rev. E 64 (2001), 036617.

Govind P. Agrawal. (2002). “Fiber Optic Communication System". Wiley-Interscience, U.S.A.

K. Senthilnathan et al. "Grating solitons near the photonic bandgap of a fiber Bragg grating," ScienceDirect, Chaos, Solitons and Fractals 33 (2007) 523531.

K. Thyagarajan and Ajoy Ghatak. (2007). "Fiber Optic Essentials". Wiley-Interscience, Canada.

K. W. Chow, Ilya M. Merhasin, Boris A. Malomed, K. Nakkeeran, K. Senthilnathan and P. K. A. Wai. "Periodic waves in fiber Bragg gratings". Physical Review E, 77, 026602 (2008).

M. Liu and P. Shum. "Simulation of soliton propagation in a directional coupler," Springer, Optical and Quantum Electronics (2006) 38:1159-1165.

Nahar Singh, Subhash C. Jain, Vandana Mishra, G. C. Poddar, Palvinder Kaur, Himani Singla, A. K. Aggrawal and Pawan Kapur. "Fibre Bragg grating - based sensing device for petrol leak detection". Current Science. Vol. 9, No. 2, January 2006.

Phing, H.S., Ali, J., Rahman, R.A., Saktioto. "Growth dynamics and characteristics of fabricated Fiber Bragg Grating using phase mask method". Microelectronics Journal. (2008).

R. Kashyap, Fiber Bragg Gratings (Academic Press, San Diego, 1999)

T. Sun, S. Pal, J. Mandal, K. T. V. Grattan. “Fibre Bragg grating fabrication uysing fluoride excimer laser for sensing and communication applications". Central Laser Facility Annual Report 2001/2002.

Yuri S. Kivshar and Govind P. Agrawal. (2003). “Optical Solitons: From Fiber to Photonics Crystal". Academic Press, U.S.A. 
Yupapin, P.P., Saktioto, T., Ali, J., Photon trapping model within a fiber bragg grating for dynamic optical tweezers use, Microwave and Optical Technology Letters 52 (4), pp. 959-961.(2010) 


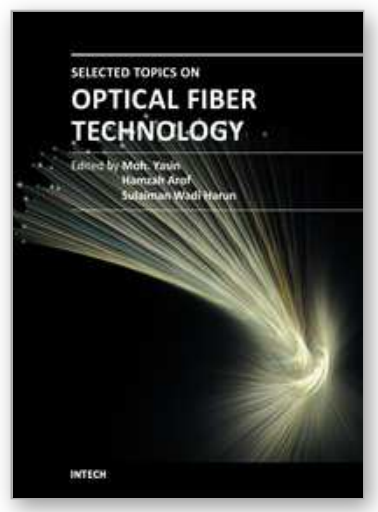

\author{
Selected Topics on Optical Fiber Technology \\ Edited by Dr Moh. Yasin
}

ISBN 978-953-51-0091-1

Hard cover, 668 pages

Publisher InTech

Published online 22, February, 2012

Published in print edition February, 2012

This book presents a comprehensive account of the recent advances and research in optical fiber technology. It covers a broad spectrum of topics in special areas of optical fiber technology. The book highlights the development of fiber lasers, optical fiber applications in medical, imaging, spectroscopy and measurement, new optical fibers and sensors. This is an essential reference for researchers working in optical fiber researches and for industrial users who need to be aware of current developments in fiber lasers, sensors and other optical fiber applications.

\title{
How to reference
}

In order to correctly reference this scholarly work, feel free to copy and paste the following:

Toto Saktioto and Jalil Ali (2012). Non Linear Optic in Fiber Bragg Grating, Selected Topics on Optical Fiber Technology, Dr Moh. Yasin (Ed.), ISBN: 978-953-51-0091-1, InTech, Available from:

http://www.intechopen.com/books/selected-topics-on-optical-fiber-technology/non-linear-optic-in-fiber-bragggrating

\section{INTECH}

open science | open minds

\section{InTech Europe}

University Campus STeP Ri

Slavka Krautzeka 83/A

51000 Rijeka, Croatia

Phone: +385 (51) 770447

Fax: +385 (51) 686166

www.intechopen.com

\section{InTech China}

Unit 405, Office Block, Hotel Equatorial Shanghai

No.65, Yan An Road (West), Shanghai, 200040, China

中国上海市延安西路65号上海国际贵都大饭店办公楼 405 单元

Phone: +86-21-62489820

Fax: +86-21-62489821 
(C) 2012 The Author(s). Licensee IntechOpen. This is an open access article distributed under the terms of the Creative Commons Attribution 3.0 License, which permits unrestricted use, distribution, and reproduction in any medium, provided the original work is properly cited. 